\title{
Robust Magnetic Tracking of Subsea Cable by AUV in the Presence of Sensor Noise and Ocean Currents
}

\author{
Caoyang Yu, Xianbo Xiang ${ }^{(0)}$, Lionel Lapierre, and Qin Zhang
}

\begin{abstract}
To inspect subsea cables, an autonomous underwater vehicle (AUV) with two triaxial magnetometers is usually assigned to track the cable route. In this paper, a novel two-layer framework synthesizing antinoise cable localization and robust tracking algorithm is proposed to guide an AUV to track subsea cables in the presence of sensor noise and ocean currents. First, an analytic formulation for the cable localization using two magnetometers is derived, and then a dedicated magnetic line-of-sight (LOS) guidance is built based on the horizontal offset between the AUV and the cable. Second, a novel antinoise method by estimating the horizontal offset is integrated into the LOS guidance law to reduce the negative effects of magnetic noise in the kinematic layer. Subsequently, in the dynamic layer, a simplified yet robust feedback controller with reduced implementation complexity is designed to track the desired guidance profiles, such that the AUV is able to track subsea cables in the presence of sensor noise and ocean currents. In addition, the capability of dynamic control laws accounting for ocean currents is analyzed in the amplitude-frequency domain. Finally, numerical studies illustrate the antinoise and robust performance of the proposed two-layer framework for subsea cable tracking.
\end{abstract}

Index Terms-Antinoise, autonomous underwater vehicles (AUVs), cable tracking, magnetic line-of-sight (LOS), robust control.

\section{INTRODUCTION}

$\mathbf{S}$ UBSEA cables provide power and communication links between continents and islands and also connect a growing number of offshore installations [1]. Yet, the cable is prone to suffer from natural disasters and human activities such as earthquake, fishing, and anchoring. Hence, it is essential to track the

Manuscript received December 16, 2016; revised June 13, 2017 and August 29, 2017; accepted October 24, 2017. Date of publication December 4, 2017; date of current version April 12,2018. This work was supported in part by the National Natural Science Foundation of China under Grant 51579111 and Grant 51209100, in part by the Shenzhen Science and Technology Plan Project under Grant JCYJ201704I311305468, in part by the Fundamental Research Funds for the Central Universities under Grant 2017KFYXJJ005, and in part by the State Key Lab Research Fund of Ocean Engineering under Grant 201504. (Corresponding author: Xianbo Xiang.)

Guest Editor: K. Asakawa.

C. Yu is with the School of Naval Architecture and Ocean Engineering, Huazhong University of Science and Technology, Wuhan 430074, China (e-mail: ycyhust@hust.edu.cn).

X. Xiang is with the School of Naval Architecture and Ocean Engineering, Huazhong University of Science and Technology, Wuhan 430074, China and also with the Shenzhen Huazhong University of Science and Technology Research Institute, Shenzhen 518057, China (e-mail: xbxiang@hust.edu.cn).

L. Lapierre is with the Department of Robotics, CNRS-Laboratoire d'Informatique, de Robotique et de Microelectronique de MontpellierUMII, UMR 5506-CC 477, Montpellier 34392 CEDEX 5, France (e-mail: lapierre@lirmm.fr).

Q. Zhang is with the State Key Laboratory of Digital Manufacturing, Equipment and Technology, Huazhong University of Science and Technology, Wuhan 430074, China (e-mail: qin.zhang@hust.edu.cn).

Digital Object Identifier 10.1109/JOE.2017.2768105 subsea cable route, which enables the inspection of the cable and its surrounding environment. To track a subsea cable, lowfrequency alternating currents (ac) are supplied to conductors in the cable, and the coaxial alternating magnetic field generated by ac can be sensed by underwater vehicles to localize the cable [2]. In fact, this electromagnetic sensing method is widely used to localize and track both uncovered and buried cables [3]. After tracking a cable route, underwater cameras and subbottom profilers carried by a remotely operated vehicle (ROV) or an autonomous underwater vehicle (AUV) can be effectively applied to inspect the present condition of the cable and the buried environment along the whole route.

Compared with an ROV demanding the support of mother vessels, AUVs are becoming the most powerful tool to support subsea pipeline inspection, marine observation and sampling, and underwater intervention [4]-[7]. For instance, dedicated AUVs for the autonomous cable tracking, such as AQUA EXPLORER 1000 and AQUA EXPLORER 2, have been developed in Japan [8], [9]. Although the operator is emancipated from the complicated operation and the operation cost is reduced because an AUV has no tether link with the mother vessel, it places greater demands on autonomy and robustness to steer the vehicle along the desired path in the presence of unknown ocean currents [2], [10].

In the cable tracking mission, the onboard controller of an AUV is required to calculate control inputs in real time so that both the relative distance and the relative direction between the AUV and the cable become zero [2], [8], [9]. Hence, in this study, the cable tracking task is innovatively formulated as a path following problem, which means an AUV is required to follow a desired geometric path along the cable route with spatial convergence, but without any time specification. However, the essential difference between the classic path following problem and the path following based cable tracking problem is that the desired path in the cable tracking problem is unknown for an AUV in prior whereas the desired path for the classic path following problem is known and parameterized [11]-[16]. In this paper, the desired path of cable tracking mission is generated online by adopting the magnetic localization method such that an AUV can follow the cable route.

In fact, there have been great efforts to develop path following controllers for AUVs in recent years. A sliding mode controller was applied to an AUV based on line-of-sight (LOS) guidance in shallow water [17]. In [18]-[20], a nonlinear path following controller by adopting the backstepping technique for an AUV was proposed. A feedback linearizing proportional-derivative 
(PD) controller combined with integral LOS guidance was proposed to achieve path following of an AUV in the presence of constant irrotational ocean currents [21]. The neural network controller and fuzzy controller for AUV tracking control have been described in [22]-[24]. These control methods have also been widely applied in unmanned surface vessels, underwater gliders, and ROVs, and the readers can refer to [25]-[34]. Nevertheless, the path following algorithms based on fuzzy logic and neural network techniques are relatively complicate and difficult to implement. Hence, a simplified yet robust algorithm is demanded for the cable tracking control system, to reduce implementation complexity of an onboard controller.

In addition, while an AUV tracks subsea cables by using the magnetic sensing method, the magnetic signal is inevitably interfered by the noise from electric motors, which drive onboard thrusters and rudders. The previous study in [35] indicated that the sensor noise will induce a spike signal in the measurement of the horizontal offset between the AUV and the cable, which likely renders jittering guidance behaviors and chattering phenomena of control inputs. The negative effects coming from sensor noise will degrade the control performance and even result in the instability of the cable tracking control system. Furthermore, it is well known that an AUV usually suffers from unknown and time-varying ocean currents while tracking the cable. In this sense, internal sensor noise and external currents disturbances make the control design of cable tracking system more difficult.

Motivated by the above considerations, a novel two-layer framework synthesizing antinoise cable localization and robust tracking algorithm is proposed. The main contributions of this paper are described as follows.

1) Compared with [2] and [35], a novel antinoise method based on magnetic localization estimation is first proposed to avoid the potential signal spike. Both graphic analysis and numerical simulation illustrate that this localization method effectively reduces the negative effects of sensor noise compared with the traditional analytic method.

2) A simplified yet robust dynamics controller is designed so that an AUV can track subsea cables in the presence of sensor noise and ocean currents. This controller reduces implementation complexity in an onboard control system. In addition, the capability of dynamic control laws accounting for ocean currents is analyzed in the amplitudefrequency domain and the whole dynamics control system is proved to be stable.

The rest of this paper is organized as follows: The nonlinear modeling of an AUV and control objectives of subsea cable tracking are presented in Section II. In Section III, the classical magnetic sensing and cable localization method are analyzed. The proposed two-layer framework composed of an antinoise magnetic LOS guidance and a robust cable tracking controller is designed in Section IV. Numerical study results are given in Section $\mathrm{V}$ to illustrate the performance of the proposed cable tracking control system, and Section VI draws the conclusions.

\section{Problem Statement}

This section defines some notations and reference frames used throughout this paper, presents the cable tracking mission,
TABLE I

NOTATIONS AND THE REFERENCE FRAMES

\begin{tabular}{ll}
\hline \hline Parameter & \multicolumn{1}{c}{ Description } \\
\hline $\begin{array}{l}\text { magnetometer 1 } \\
\text { magnetometer 2 }\end{array}$ & $\begin{array}{l}\text { The larboard triaxial magnetometer } \\
\text { The starboard triaxial magnetometer } \\
\text { AUV }\end{array}$ \\
A OS & Line of sight \\
SNR & Signal-to-noise ratio \\
$\psi_{B r}$ & The heading deviation \\
$\psi_{e}$ & The course deviation \\
$Y$ & The horizontal offset \\
$Z$ & The vertical distance \\
$\left(A_{*}, B_{*}, C_{*}\right)$ & The magnetic field \\
$\{I\}$ & The earth-fixed inertial frame \\
$\{B\}$ & The body-fixed frame of an AUV \\
\hline \hline
\end{tabular}

describes the kinematic and dynamic equations of an AUV in the fixed-depth plane, and formulates the problem of subsea cable tracking.

\section{A. Definition}

To explicitly introduce the cable tracking mission, the notations and reference frames in Table I will be used throughout this paper.

\section{B. Cable Tracking Mission}

As stated in [8], the cable tracking mission is to reduce the relative direction and the relative distance between an AUV and subsea cables to be zero. To accomplish the mission, two triaxial magnetometers are installed in two wide wings of an AUV to localize the cable. Assuming that this AUV is equipped with a stern propeller and two pairs of stern rudders, it has no thrusters to directly contribute the corresponding forces and moment in sway, heave, and roll and belongs to an underactuated system. In this paper, the roll and pitch motions of this underactuated AUV are ignored in consideration of an enough large metacentric height and relatively large contact area of two wide wings in the sea.

During the cable tracking mission, an AUV first dives to a suitable height relative to the seabed and then moves in a given direction obtained from the cable laying report, as shown in Fig. 1 , where $\left(A_{1}, B_{1}, C_{1}\right)$ and $\left(A_{2}, B_{2}, C_{2}\right)$ are the magnetic fields in three axes of magnetometer 1 and magnetometer 2, respectively. In this paper, the vertical distance $Z$ between the AUV and the cable is assumed to be $2 \mathrm{~m}$. In this sense, we focus on how to control the surge speed and heading of the underactuated AUV to track the cable route in the presence of sensor noise and time-varying ocean currents.

\section{Nonlinear AUV Model}

According to [36], an AUV moving in the horizontal plane can be represented by three-degrees-of-freedom components in the surge, sway, and yaw directions. Hence, the planar kinematic equations of an AUV can take the form

$$
\left\{\begin{array}{l}
\dot{x}=u \cos (\psi)-v \sin (\psi) \\
\dot{y}=u \sin (\psi)+v \cos (\psi) \\
\dot{\psi}=r
\end{array}\right.
$$




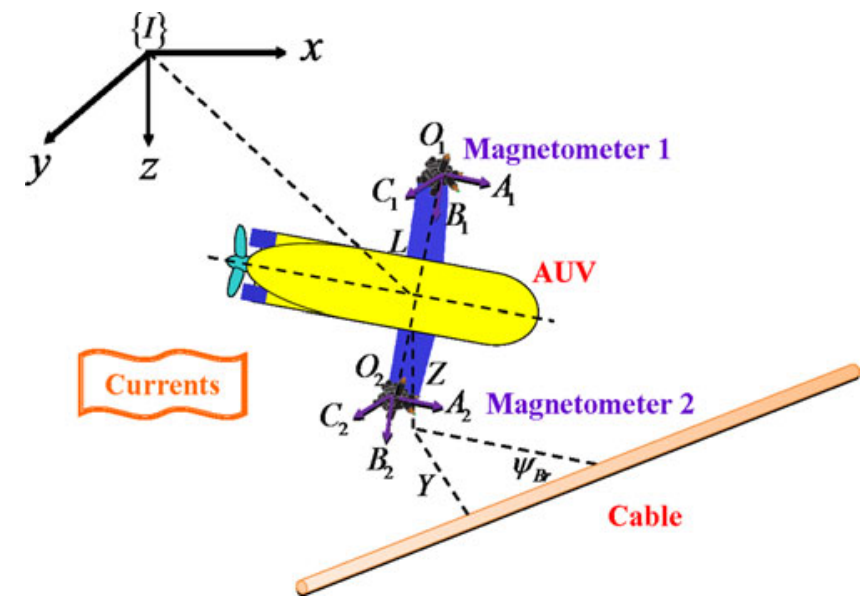

Fig. 1. Magnetic sensing and cable tracking.

where $(x, y, \psi)$ denotes the position and heading of the AUV with respect to the frame $\{I\}$, and $(u, v, r)$ denotes the surge speed, sway speed, and yaw speed in the frame $\{B\}$, respectively. According to [37], the dynamic equations of an AUV moving in the horizontal plane can be described as

$$
\left\{\begin{aligned}
\dot{u}= & \frac{m_{22}}{m_{11}} v r-\frac{d_{11}}{m_{11}} u-\sum_{i=2}^{3} \frac{d_{u i}}{m_{11}}|u|^{i-1} u+\frac{\tau_{u}}{m_{11}} \\
& +\frac{\tau_{E u}}{m_{11}} \\
\dot{v}= & -\frac{m_{11}}{m_{22}} u r-\frac{d_{22}}{m_{22}} v-\sum_{i=2}^{3} \frac{d_{v i}}{m_{22}}|v|^{i-1} v+\frac{\tau_{E v}}{m_{22}} \\
\dot{r}= & \frac{m_{11}-m_{22}}{m_{33}} u v-\frac{d_{33}}{m_{33}} r-\sum_{i=2}^{3} \frac{d_{r i}}{m_{33}}|r|^{i-1} r+\frac{\tau_{r}}{m_{33}} \\
& +\frac{\tau_{E r}}{m_{33}}
\end{aligned}\right.
$$

where $m_{(\cdot)}$ expresses the hydrodynamic derivatives of the AUV and $d_{(\cdot)}$ captures its hydrodynamic damping effects. In addition, $\left(\tau_{u}, \tau_{r}\right)$ denotes the propeller thrust and the yaw moment represented in the frame $\{B\} .\left(\tau_{E u}, \tau_{E v}, \tau_{E r}\right)$ denotes the ocean current disturbances acting in surge, sway and yaw, respectively.

\section{Control Objective}

As previously mentioned, the cable tracking mission essentially can be regarded as a classic path following problem. Hence, for a straight subsea cable shown in Fig. 2, the problem of cable tracking control for an AUV exposed to sensor noise and unknown ocean currents can be formulated by

$$
\left\{\begin{array}{l}
\lim _{t \rightarrow \infty} Y=0 \\
\lim _{t \rightarrow \infty} \psi_{e}=0
\end{array}\right.
$$

where $\psi_{e}=\psi_{w}-\psi_{r}$ is the course deviation between the course angle $\psi_{w}$ of the AUV and the direction $\psi_{r}$ of the cable. Note that the first term in (3) demands that the horizontal offset between the AUV and the cable converge to zero, and the second term requires that the resultant speed of the AUV be aligned with the tangent of the cable.

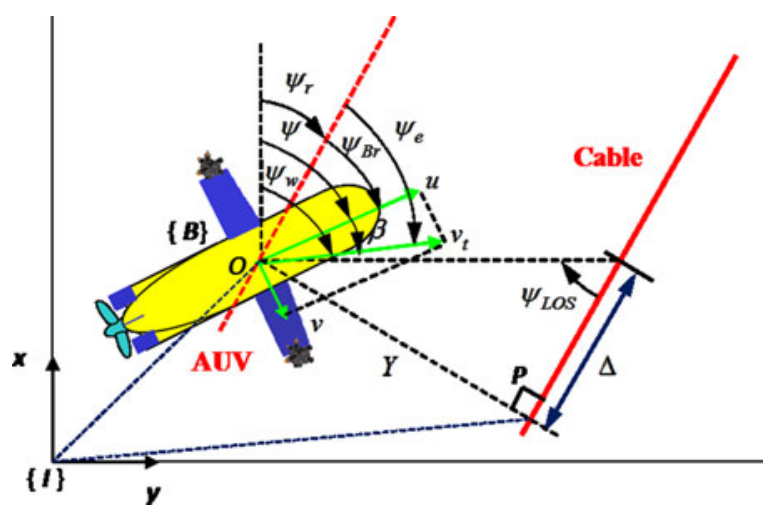

Fig. 2. Cable tracking control.

\section{Magnetic Sensing}

In this section, an analytic method of magnetic cable localization including the course deviation and the horizontal offset is formulated. Subsequently, the negative effects resulted by sensor noise are analyzed, which inspires the design of antinoise magnetic LOS guidance law in the kinematic layer.

\section{A. Magnetic Field}

Suppose that the straight cable tracked by an AUV is parameterized by

$$
\left\{\begin{array}{l}
y_{r}=k x_{r}+b \\
z_{r}=c
\end{array}\right.
$$

where $\left(x_{r}, y_{r}, z_{r}\right)$ is the position of the cable in the frame $\{I\}, k$ is the slope of the cable horizontal-plane projection in the frame $\{I\}$, and $b$ and $c$ are constants.

Let $(x, y, z)$ be the position of an AUV with respect to the frame $\{I\}$. According to [35], the magnetic fields $\left(A_{i}, B_{i}, C_{i}\right)$ of magnetometer $i$ with $i=1,2$ can be described as

$$
\begin{aligned}
{\left[\begin{array}{c}
A_{1} \\
B_{1} \\
C_{1}
\end{array}\right]=} & {\left[\begin{array}{c}
\frac{k_{\varepsilon}\left(z_{r}-z\right)}{R_{1}^{2}} \sin (\psi-\arctan (k)) \\
\frac{k_{\varepsilon}\left(z_{r}-z\right)}{R_{1}^{2}} \cos (\psi-\arctan (k)) \\
\frac{k_{\varepsilon}}{R_{1}^{2}}-k\left(x+\frac{L}{2} \sin (\psi)\right)+\left(y-\frac{L}{2} \cos (\psi)\right)-b \\
\sqrt{k^{2}+1}
\end{array}\right] } \\
& +f_{\text {noi } 1}
\end{aligned}
$$

and

$$
\begin{aligned}
& {\left[\begin{array}{l}
A_{2} \\
B_{2} \\
C_{2}
\end{array}\right]=} {\left[\begin{array}{c}
\frac{k_{\varepsilon}\left(z_{r}-z\right)}{R_{2}^{2}} \sin (\psi-\arctan (k)) \\
\frac{k_{\varepsilon}\left(z_{r}-z\right)}{R_{2}^{2}} \cos (\psi-\arctan (k)) \\
\frac{k_{\varepsilon}}{R_{2}^{2}}-k\left(x-\frac{L}{2} \sin (\psi)\right)+\left(y+\frac{L}{2} \cos (\psi)\right)-b \\
\sqrt{k^{2}+1}
\end{array}\right] } \\
&+f_{\text {noi } 2}
\end{aligned}
$$




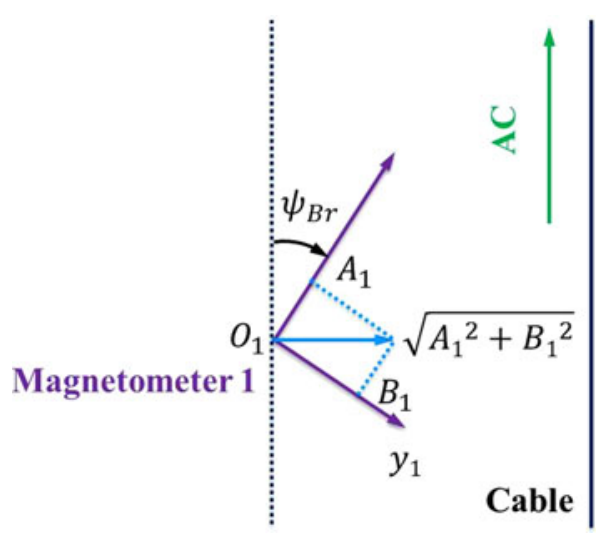

Fig. 3. Heading deviation calculation.

where $k_{\varepsilon}$ is the constant and depends on the ac in the cable, $L$ is the spacing between magnetometer 1 and magnetometer 2

$R_{1}=\sqrt{\frac{\left(k\left(x+\frac{L}{2} \sin (\psi)-\left(y-\frac{L}{2} \cos (\psi)\right)+b\right)^{2}\right.}{k^{2}+1}+\left(z_{r}-z\right)^{2}}$

represents the distance between magnetometer 1 and the cable, and

$R_{2}=\sqrt{\frac{\left(k\left(x-\frac{L}{2} \sin (\psi)-\left(y+\frac{L}{2} \cos (\psi)\right)+b\right)^{2}\right.}{k^{2}+1}+\left(z_{r}-z\right)^{2}}$

represents the distance between magnetometer 2 and the cable. In addition, $f_{\text {noi1 }}$ and $f_{\text {noi2 }}$ denote the sensor noise on magnetometer 1 and magnetometer 2, respectively.

Remark 1: For cable tracking, the typical ac following in subsea cables is about $0.1 \mathrm{~A}$, which makes the magnetic field $10 \mathrm{nT}$ at the altitude of $2 \mathrm{~m}$ [8]. Based on this, $k_{\varepsilon}$ can be set as $2 \times 10^{-8}$.

\section{B. Cable Localization}

1) Course Deviation: In this paper, assume that the cable is located on the right-hand side of two magnetometers. The body axes $O_{i} x_{i}, O_{i} y_{i}$, and $O_{i} z_{i}$ coincide with the principal axes of inertia of magnetometer $i$ and are defined as follows: $O_{i} x_{i}$ is the longitudinal axis directed from aft to fore of magnetometer $i, O_{i} y_{i}$ is the transverse axis directed from port side to starboard of magnetometer $i$, and $O_{i} z_{i}$ is the normal axis directed from top to down of magnetometer $i$. The magnetic fields projected in the horizontal plane are depicted in Fig. 3, where the heading deviation $\psi_{B r}$ between the heading angle $\psi$ of an AUV and the direction $\psi_{r}$ of subsea cables can be calculated by

$$
\psi_{B r}=\operatorname{atan} 2\left(A_{1}, B_{1}\right)
$$

where the function atan2 is the arctangent function with two arguments, which ensures the mapping $\psi_{B r} \in(-\pi, \pi]$.

From Fig. 2, the course deviation $\psi_{e}$ is

$$
\psi_{e}=\psi_{B r}+\beta
$$

where the side-slip angle $\beta$ is defined as

$$
\beta=\arctan \left(\frac{v}{u}\right)
$$

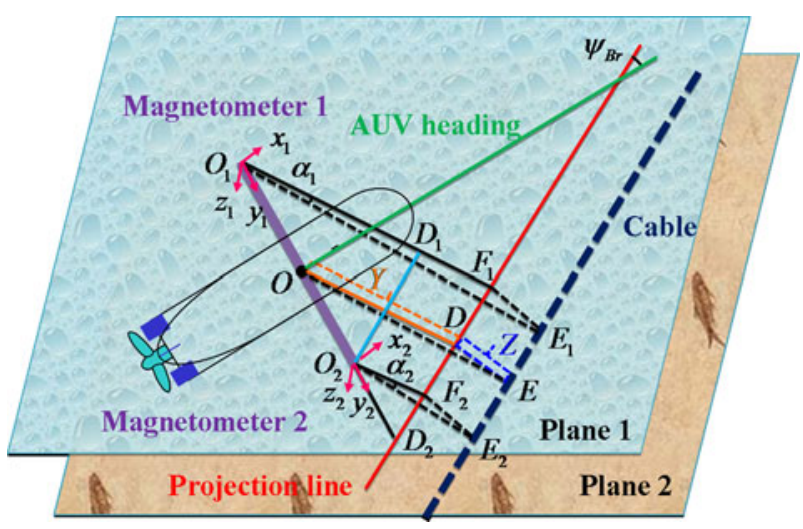

Fig. 4. Subsea cable localization.

Similarly, the same course deviation result can be obtained while the cable is located on the left-hand side or in the middle of two magnetometers.

2) Horizontal Offset: As shown in Fig. 4, the onboard triaxial magnetometers and the blue-black cable are located in plane 1 and plane 2, respectively. In plane 1, the red dashed line denotes the projection line of the cable, and the green dashed line denotes the AUV heading. The purple line connects the center $O_{1}$ of magnetometer 1 and the center $O_{2}$ of magnetometer 2. Constructing the auxiliary lines as $\overrightarrow{O_{1} E_{1}} \perp \overrightarrow{E_{1} E_{2}}$, $\overrightarrow{E_{1} F_{1}} \perp \overrightarrow{O_{1} F_{1}}, \overrightarrow{O_{2} E_{2}} \perp \overrightarrow{E_{1} E_{2}}, \overrightarrow{E_{2} F_{2}} \perp \overrightarrow{O_{2} F_{2}}$, and $\overrightarrow{O_{2} D_{1}} \perp \overrightarrow{O_{1} D_{1}}$ gives the following geometrical relationships to localize the cable: $\overline{F_{1} E_{1}}=\overline{F_{2} E_{2}}, \triangle O_{1} D_{2} F_{1} \sim \triangle O_{2} D_{2} F_{2}, \overline{F_{1} D_{1}}=\overline{O_{2} F_{2}}$, and $\angle O_{2} O_{1} D_{1}=\psi_{B r}$, where $\overline{P_{1} P_{2}}$ denotes the norm of vector $\overrightarrow{P_{1} P_{2}}, \triangle$ and $\angle$ are the symbols of triangle and angle, and $\sim$ connects two homothetic triangles.

Since $\triangle O_{1} D_{2} F_{1} \sim \triangle O_{2} D_{2} F_{2}$, it renders

$$
\frac{\overline{O_{1} D_{2}}}{\overline{O_{2} D_{2}}}=\frac{\overline{O_{1} F_{1}}}{\overline{O_{2} F_{2}}} .
$$

Considering the triangles $\triangle O_{1} F_{1} E_{1}$ and $\triangle O_{2} F_{2} E_{2}$, it has

$$
\left\{\begin{array}{l}
\overline{O_{1} F_{1}}=\frac{\overline{F_{1} E_{1}}}{\tan \left(\alpha_{1}\right)} \\
\overline{O_{2} F_{2}}=\frac{\overline{F_{2} E_{2}}}{\tan \left(\alpha_{2}\right)} .
\end{array}\right.
$$

Substituting (11) into (10) leads to

$$
\overline{\overline{O_{1} D_{2}}}=\frac{\tan \left(\alpha_{2}\right)}{\tan \left(\alpha_{1}\right)} .
$$

As shown in Fig. 5, there is

$$
\left\{\begin{array}{l}
\tan \left(\alpha_{1}\right)=-\frac{\sqrt{A_{1}^{2}+B_{1}^{2}}}{C_{1}} \\
\tan \left(\alpha_{2}\right)=-\frac{\sqrt{A_{2}^{2}+B_{2}^{2}}}{C_{2}} .
\end{array}\right.
$$




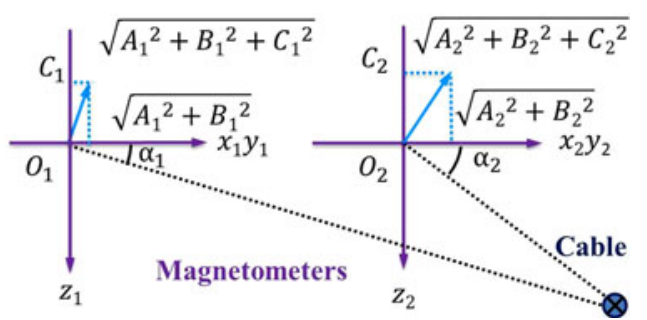

Fig. 5. Elevation view in the direction of input currents.

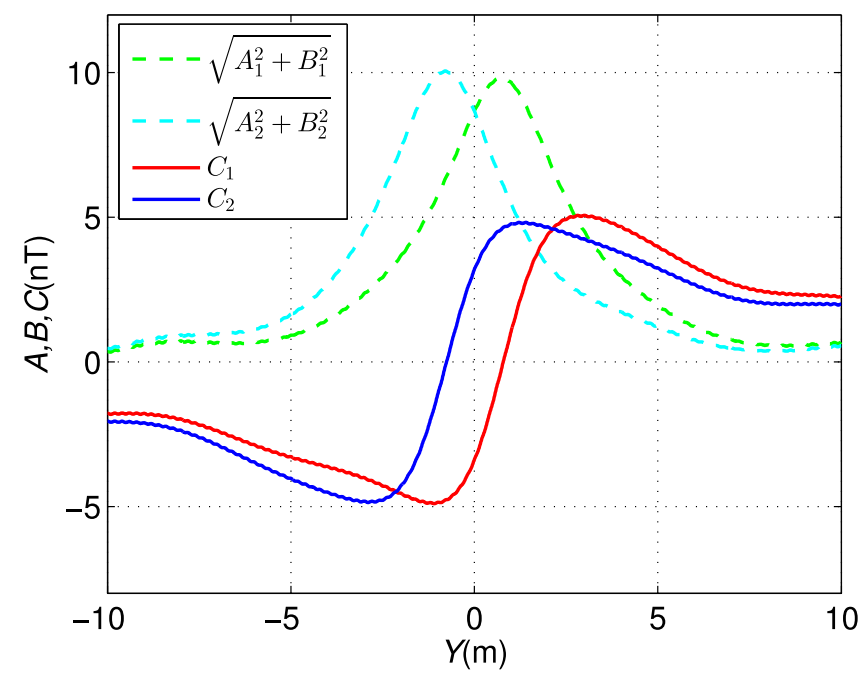

Fig. 6. Transitions of the magnetic fields on two magnetometers.

Combining the following geometrical relationships:

$$
\left\{\begin{array}{l}
\overline{O_{1} D_{2}}=\overline{O D_{2}}+\overline{O O_{1}} \\
\overline{O_{2} D_{2}}=\overline{O D_{2}}-\overline{O O_{2}} \\
\overline{O O_{1}}=\overline{O O_{2}}
\end{array}\right.
$$

with (12) and (13) yields

$$
\overline{O D_{2}}=\frac{C_{1} \sqrt{A_{2}^{2}+B_{2}^{2}}+C_{2} \sqrt{A_{1}^{2}+B_{1}^{2}}}{C_{1} \sqrt{A_{2}^{2}+B_{2}^{2}}-C_{2} \sqrt{A_{1}^{2}+B_{1}^{2}}} \frac{L}{2} .
$$

According to Fig. 4, the horizontal offset $Y$ between the AUV and the cable can be calculated as

$$
Y=\frac{C_{1} \sqrt{A_{2}^{2}+B_{2}^{2}}+C_{2} \sqrt{A_{1}^{2}+B_{1}^{2}}}{-C_{1} \sqrt{A_{2}^{2}+B_{2}^{2}}+C_{2} \sqrt{A_{1}^{2}+B_{1}^{2}}} \frac{L \cos \left(\psi_{B r}\right)}{2} .
$$

Similarly, the same horizontal offset result can be obtained while the cable is located on the left-hand side or in the middle of two magnetometers.

\section{Noise Analysis}

Assume that the heading deviation $\psi_{B r}$ is zero. In this case, when an AUV exposed to sensor noise moves from the lefthand side of subsea cables to the right-hand side, the transitions of $\sqrt{A_{1}^{2}+B_{1}^{2}}, \sqrt{A_{2}^{2}+B_{2}^{2}}, C_{1}$, and $C_{2}$ in (16) are described in Fig. 6. According to (16), the transitions of the corresponding measured horizontal offset from magnetometer readings are

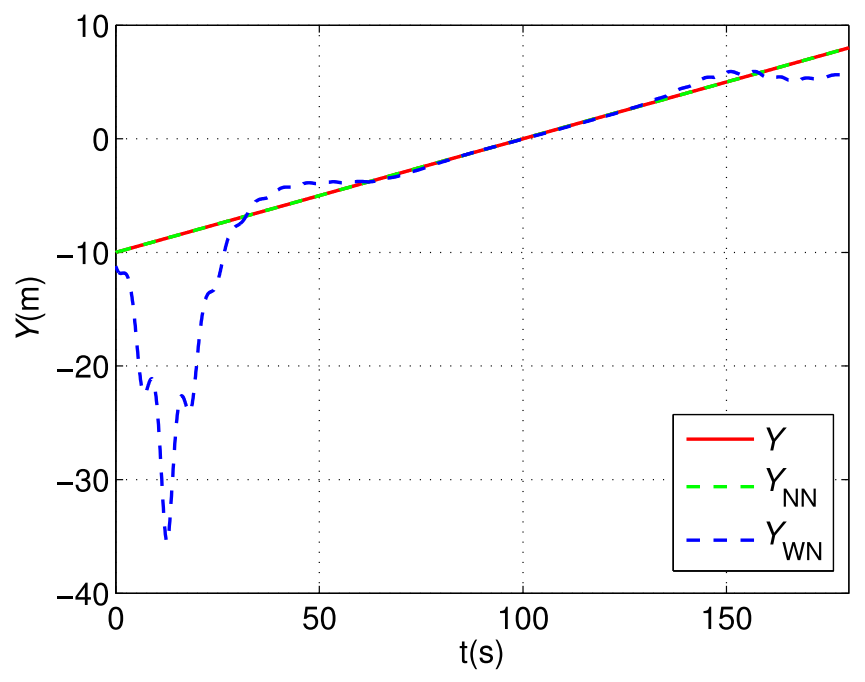

Fig. 7. Transitions of the measured horizontal offset.

shown in Fig. 7, where the measured horizontal offset (green dotted line) without sensor noise is identical with the actual horizontal offset (red line).

However, a spike signal is induced in the measurement of the horizontal offset (blue dotted line) when the AUV approaches the cable from far away in the presence of sensor noise. The reason is that the signal amplitude is small, as shown in Fig. 6, which will lead to a small signal-to-noise ratio (SNR). In this sense, the small signal amplitude is prone to result in the singularity where the denominator in (16) tends to zero. In the cable tracking mission, this spike signal likely renders jittering guidance behaviors and chattering phenomena of control inputs. Therefore, the negative effects of sensor noise will degrade the control performance and even result in the instability of cable tracking control system. In this paper, this problem will be solved by introducing a saturated LOS guidance law integrated with antinoise magnetic localization estimation.

\section{Controller Design}

In this section, a saturated LOS guidance law integrated with antinoise magnetic localization estimation is proposed to reduce the negative effects of sensor noise in the kinematic layer, and then a simplified yet robust feedback linearizing proportionalintegral-derivative (PID) tracking controller with reduced implementation complexity is designed to reject ocean current disturbances in the dynamic layer.

\section{A. Magnetic LOS Guidance}

LOS is a popular course guidance law applied in the marine vehicle system [14], [20], [36], [38]. In this paper, magnetic LOS guidance is proposed and built in the projection line of subsea cables. As shown in Fig. 2, the magnetic LOS guidance angle is defined as

$$
\psi_{\mathrm{LOS}}=-\arctan \left(\frac{Y}{\Delta}\right)
$$


where $\Delta$ is the look ahead distance and is usually equal to two vehicles' length. Obviously, the magnetic LOS guidance law is equivalent to a saturated proportional control law, effectively mapping the horizontal offset $Y \in \mathbb{R}$ into $\psi_{\text {LOS }} \in(-\pi / 2, \pi / 2)$. Note that $Y$ cannot be directly solved based on the coordinate information as shown in [14], [20], and [36] because the cable route is unknown for an AUV in prior. While in the magnetic LOS algorithm, $Y$ is indirectly calculated through magnetic sensing.

According to the LOS guidance principle [14], [18], [20], if the course deviation $\psi_{e}$ is driven to be equal to $\psi_{\mathrm{LOS}}$, the AUV will move toward and then follow the cable with imitated helmsman behaviors.

\section{B. Kinematic Controller}

As control objectives of subsea cable tracking are to drive the horizontal offset $Y$ and the course deviation $\psi_{e}$ to be zero, the corresponding positive-definite Lyapunov function candidate can be designed as

$$
V=\frac{1}{2} Y^{2}+\frac{1}{2}\left(\psi_{e}-\psi_{\mathrm{LOS}}\right)^{2}
$$

The derivative of $V$ is

$$
\dot{V}=v_{t} Y \sin \left(\psi_{e}\right)+\left(\psi_{e}-\psi_{\mathrm{LOS}}\right)\left(\dot{\psi}_{e}-\dot{\psi}_{\mathrm{LOS}}\right)
$$

with the resultant speed $v_{t}=\sqrt{u^{2}+v^{2}}>0$.

Similar to [20], it is straightforward to choose the kinematic control law as

$$
\dot{\psi}_{e}=\dot{\psi}_{\mathrm{LOS}}-k_{\psi}\left(\psi_{e}-\psi_{\mathrm{LOS}}\right)-v_{t} Y f\left(\psi_{e}, \psi_{\mathrm{LOS}}\right)
$$

to let the derivative of $V$ be negative definite. Here, $k_{\psi}$ is a positive gain and

$$
f\left(\psi_{e}, \psi_{\mathrm{LOS}}\right)=\frac{\sin \left(\psi_{e}\right)-\sin \left(\psi_{\mathrm{LOS}}\right)}{\psi_{e}-\psi_{\mathrm{LOS}}} .
$$

Remark 2: $f\left(\psi_{e}, \psi_{\text {LOS }}\right)$ is a nonsingular and continuous function because

$$
f\left(\psi_{e}, \psi_{\mathrm{LOS}}\right)=\cos \left(\frac{\psi_{e}+\psi_{\mathrm{LOS}}}{2}\right) \frac{\sin \left(\frac{\psi_{e}-\psi_{\mathrm{LOS}}}{2}\right)}{\frac{\psi_{e}-\psi_{\mathrm{LOS}}}{2}}
$$

and

$$
\lim _{\psi_{e} \rightarrow \psi_{\mathrm{LOS}}} f\left(\psi_{e}, \psi_{\mathrm{LOS}}\right)=\cos \left(\psi_{e}\right)
$$

Substituting (20) into (19) leads to

$$
\dot{V}=-\frac{v_{t} Y^{2}}{\sqrt{Y^{2}+\Delta^{2}}}-k_{\psi}\left(\psi_{e}-\psi_{\mathrm{LOS}}\right)^{2} \leq 0 .
$$

According to the Lyapunov stability theorem, it is concluded that

$$
\lim _{t \rightarrow \infty}\left(Y, \psi_{e}-\psi_{\mathrm{LOS}}\right)=(0,0)
$$

Obviously, $\lim _{t \rightarrow \infty} Y=0$ means that $\lim _{t \rightarrow \infty} \psi_{\text {LOS }}=0$. Hence, under the kinematic control law (20)

$$
\lim _{t \rightarrow \infty}\left(Y, \psi_{e}\right)=(0,0) \text {. }
$$

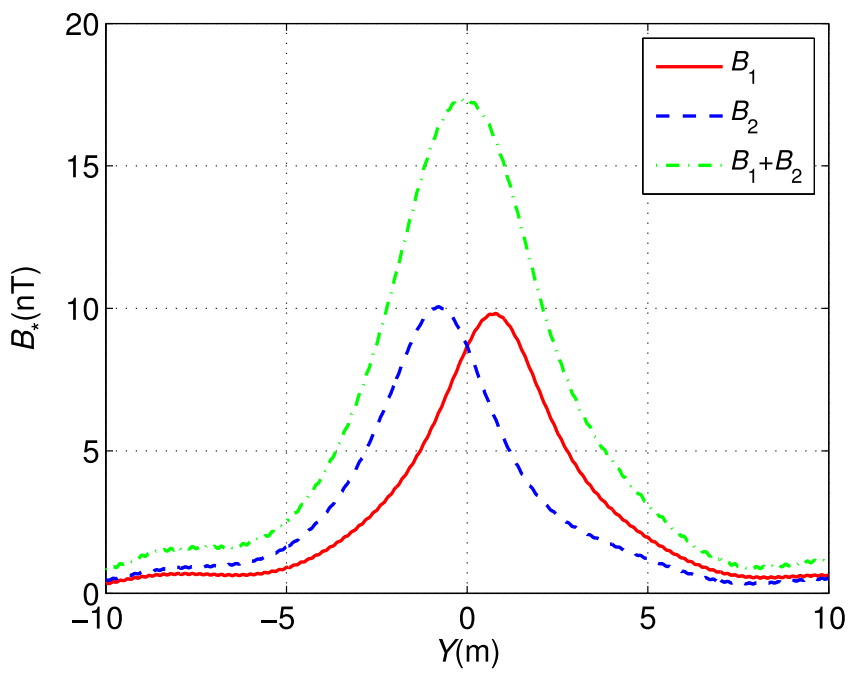

Fig. 8. Transitions of magnetic fields on two magnetometers.

TABLE II

RELATIONSHIP BETWEEN MAGNETIC Signal AND Horizontal OFFSET

\begin{tabular}{lccc}
\hline \hline Term & Left & Middle & Right \\
\hline Magnetic signal & $B_{1}>B_{2}$ & $B_{1}=B_{2}$ & $B_{1}<B_{2}$ \\
Horizontal offset & $Y>0$ & $Y=0$ & $Y<0$ \\
\hline \hline
\end{tabular}

Remark 3: Although the saturated LOS guidance angle in (17) can reduce the negative effects of a spike signal in the measurement of the horizontal offset $Y, Y$ also appears as a multiplier in the kinematic control law (20), which likely also renders jittering guidance behaviors in the presence of sensor noise. Below is an antinoise method for calculating the horizontal offset based on magnetic localization estimation, which can prevent the potential measurement spike from generating when an AUV approaches subsea cables from far away.

\section{Antinoise Magnetic Estimation}

Based on the assumptions in Section III-C, the transitions of the magnetic fields along the $y$-axis of magnetometers and their sum are described in Fig. 8. Obviously, when the cable is located on the right-hand side of the middle point between two magnetometers, the horizontal offset is negative and the magnetic field along the $y$-axis of magnetometer 1 is smaller than that of magnetometer 2 . When the cable is located below the middle point, the horizontal offset becomes zero and the magnetic field along the $y$-axis of magnetometer 1 is identical to that of magnetometer 2 . When the cable is located on the lefthand side of the middle point, the horizontal offset is positive and the magnetic field along the $y$-axis of magnetometer 1 is larger than that of magnetometer 2. In addition, the closer the distance between the AUV and the cable, the stronger the sum of magnetic flux density along the $y$-axis of magnetometers.

The above results can be summarized in Table II, where the sign of the horizontal offset $Y$ depends on the sign of the magnetic signal difference $\left(B_{1}-B_{2}\right)$. As shown in Fig. 9, the transi- 


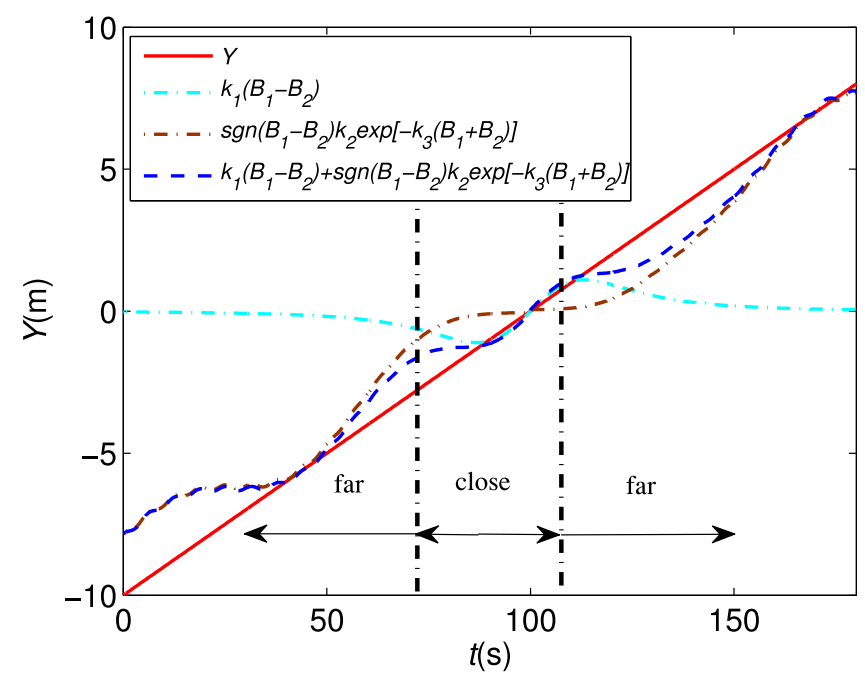

Fig. 9. Transitions of the estimated horizontal offset components.

tion of $\left(B_{1}-B_{2}\right)$ agrees well with that of the horizontal offset $Y$ when $Y$ is relatively small. Yet, $\left(B_{1}-B_{2}\right)$ quickly converges to zero when $Y$ becomes large. Combining Fig. 8 with Fig. 9, a negative exponential function with respect to the sum $\left(B_{1}+B_{2}\right)$ can be introduced to estimate the case of a large horizontal offset. Note that $\left(B_{1}+B_{2}\right)$ becomes small and the negative exponential function quickly increases when $Y$ is large, whereas $\left(B_{1}+B_{2}\right)$ becomes large and the negative exponential function tends to zero when $Y$ is close to zero.

Based on the above analysis, it is possible to estimate the horizontal offset by using a simplified nonlinear equation as

$$
Y=k_{1}\left(B_{1}-B_{2}\right)+\operatorname{sgn}\left(B_{1}-B_{2}\right) k_{2} e^{-k_{3}\left(B_{1}+B_{2}\right)}
$$

with positive control gains $k_{1}, k_{2}, k_{3}$ and a sign function sgn. As shown in Fig. 9, the blue line that represents the estimated horizontal offset is basically consistent with the actual horizontal offset in the presence of sensor noise. Although there are still few differences, they converge to zero when the AUV is located above the cable. Hence, as long as the horizontal offset $Y$ estimated from the magnetometer readings converge to zero, it can be guaranteed that the AUV is located above the cable route.

Remark 4: Formulation (26) estimated from the magnetometer readings is different from the nominal value given in [2, eq. (16)] and [35, eq. (16)], which depends on the accurate, complicated math deduction. Obviously, this improved formulation (26) has no fraction, which is instrumental in reducing the negative effects of sensor noise and preventing the potential spike from generating.

\section{Dynamic Controller}

Since $\psi_{e}=\psi_{w}-\psi_{r}$, the error rate between the course angle $\psi_{w}$ of the AUV and the direction $\psi_{r}$ of subsea cables is

$$
\dot{\psi}_{e}=r_{d}+\dot{\beta}-\dot{\psi}_{r}
$$

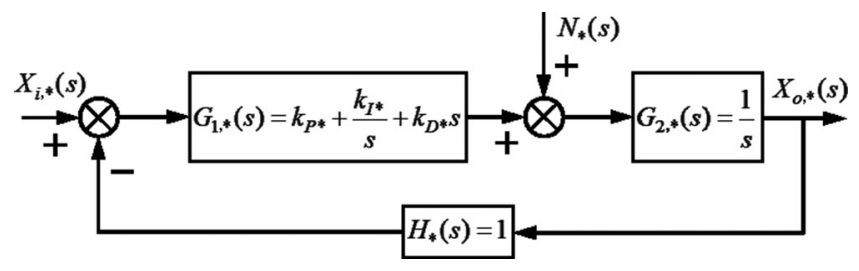

Fig. 10. Block diagram of the dynamic control system.

Assume that subsea cables tracked by the AUV are straight, which means that $\psi_{r}$ is constant and its derivative $\dot{\psi}_{r}$ is zero. Substituting (20) into (27) leads to the reference yaw speed $r_{d}$ as

$r_{d}=\dot{\psi}_{\mathrm{LOS}}-k_{\psi}\left(\psi_{e}-\psi_{\mathrm{LOS}}\right)-v_{t} Y \frac{\sin \left(\psi_{e}\right)-\sin \left(\psi_{\mathrm{LOS}}\right)}{\psi_{e}-\psi_{\mathrm{LOS}}}-\dot{\beta}$

where $\psi_{\mathrm{LOS}}, \psi_{e}$, and $\beta$ are defined in (17), (8), and (9), respectively.

In the cable tracking mission, an AUV is usually demanded a constant reference surge speed $u_{d}$. Hence, the speed errors in the dynamic layer can be summarized as

$$
\left\{\begin{array}{l}
e_{u}=u_{d}-u \\
e_{r}=r_{d}-r
\end{array}\right.
$$

To reduce the errors (29) to be zero, the dynamic control laws that act on the surge and yaw directions of an AUV can be designed as

$$
\left\{\begin{aligned}
\tau_{u}= & -m_{22} v r+d_{11} u+\sum_{i=2}^{3} d_{u i}|u|^{i-1} u \\
& +m_{11}\left(k_{P u} e_{u}+k_{I u} \int_{0}^{t} e_{u} d \tau+k_{D u} \dot{e}_{u}\right) \\
\tau_{r}= & -\left(m_{11}-m_{22}\right) u v+d_{33} r+\sum_{i=2}^{3} d_{r i}|r|^{i-1} r \\
& +m_{33}\left(k_{P r} e_{r}+k_{I r} \int_{0}^{t} e_{r} d \tau+k_{D r} \dot{e}_{r}\right) .
\end{aligned}\right.
$$

Here, the feedback linearizing technique is first adopted to eliminate the unknown system nonlinear terms, and then a PID algorithm with reduced implementation complexity is designed to reject unknown current disturbances.

Substituting (30) into (2) leads to

$$
\left\{\begin{array}{l}
\dot{u}=\left(k_{P u} e_{u}+k_{I u} \int_{0}^{t} e_{u} d \tau+k_{D u} \dot{e}_{u}\right)+\frac{\tau_{E u}}{m_{11}} \\
\dot{r}=\left(k_{P r} e_{r}+k_{I r} \int_{0}^{t} e_{r} d \tau+k_{D r} \dot{e}_{r}\right)+\frac{\tau_{E r}}{m_{33}} .
\end{array}\right.
$$

Assume that the Laplace transform of the control input $u_{d}(t), r_{d}(t)$ is $X_{i, *}(s)$ with $* \in\{u, r\}$, the Laplace transform of the output $u(t), r(t)$ is $X_{o, *}(s)$, and the Laplace transform of the current disturbance $\left(\tau_{E u} / m_{11}\right)(t),\left(\tau_{E r} / m_{33}\right)(t)$ is $N_{*}(s)$. According to [39], the Laplace transform of (31) can be assembled into

$$
\begin{aligned}
s X_{o, *}(s)= & k_{P *}\left(X_{i, *}(s)-X_{o, *}(s)\right)+k_{I *} \frac{X_{i, *}(s)-X_{o, *}(s)}{s} \\
& +k_{D *} s\left(X_{i, *}(s)-X_{o, *}(s)\right)+N_{*}(s) .
\end{aligned}
$$

Hence, the block diagram of the dynamic controller can be depicted in Fig. 10. Obviously, this controller consists of a proportional term, an integral term, and a derivative term. 
The closed-loop transfer function $G_{X_{i, *}}(s)$ under the control input of $X_{i, *}(s)$ is given by

$$
G_{X_{i, *}}(s)=\frac{X_{o 1, *}(s)}{X_{i, *}(s)}=\frac{k_{D *} s^{2}+k_{P *} s+k_{I *}}{\left(k_{D *}+1\right) s^{2}+k_{P *} s+k_{I *}}
$$

where $X_{o 1, *}(s)$ is the Laplace output resulting from the input $X_{i, *}(s)$ as $N_{*}(s)=0$.

The closed-loop transfer function $G_{N_{*}}(s)$ under the noise input of $N_{*}(s)$ is

$$
G_{N_{*}}(s)=\frac{X_{o 2, *}(s)}{N_{*}(s)}=\frac{s}{\left(k_{D *}+1\right) s^{2}+k_{P *} s+k_{I *}}
$$

where $X_{o 2, *}(s)$ is the Laplace output resulting from the current disturbance $N_{*}(s)$ as $X_{i, *}(s)=0$.

When both the input $X_{i, *}(s)$ and the current disturbance $N_{*}(s)$ act on the dynamic system, the output of the whole system is

$$
\begin{aligned}
X_{o, *}(s)= & X_{o 1, *}(s)+X_{o 2, *}(s) \\
= & \frac{k_{D *} s^{2}+k_{P *} s+k_{I *}}{\left(k_{D *}+1\right) s^{2}+k_{P *} s+k_{I *}} X_{i, *}(s) \\
& +\frac{s}{\left(k_{D *}+1\right) s^{2}+k_{P *} s+k_{I *}} N_{*}(s) \\
= & \frac{\left(k_{D *} s^{2}+k_{P *} s+k_{I *}\right) X_{i, *}(s)+s N_{*}(s)}{\left(k_{D *}+1\right) s^{2}+k_{P *} s+k_{I *}} .
\end{aligned}
$$

Remark 5: $k_{P *}>0, k_{I *}>0$, and $k_{D *}>0$ guarantee that all the characteristic roots of the dynamic control system have negative real parts. Hence, the dynamic control system is asymptotically stable, namely $\lim _{t \rightarrow \infty} x_{o 1, *}(t)=x_{i, *}(t)$ and $\lim _{t \rightarrow \infty} x_{o 2, *}(t)=0$, which indicates that the AUV is capable of rejecting unknown ocean current disturbances and then let the steerable surge and yaw speeds converge to the respective desired profile.

The transfer function (34) yields that

$$
\begin{aligned}
X_{o 2, *}(s) & =\frac{1}{\left(k_{D *}+1\right) s+\frac{k_{I *}}{s}+k_{P *}} N_{*}(s) \\
& =\frac{1}{\delta_{*}(s)} N_{*}(s)
\end{aligned}
$$

with $\delta_{*}(s)=\left(k_{D *}+1\right) s+\frac{k_{I *}}{s}+k_{P *}$, where the amplitudefrequency characteristic of $\delta(s)$ is

$$
A_{*}(\omega)=\left|\delta_{*}(j \omega)\right|=\sqrt{k_{P *}^{2}+\left[\left(k_{D *}+1\right) \omega-\frac{k_{I *}}{\omega}\right]^{2}}
$$

with $j=\sqrt{-1}$ denoting the imaginary unit. The corresponding evolution of amplitude-frequency characteristic with respect to the frequency $\omega$ is shown in Fig. 11. Obviously, it has a minimum value of $k_{P *}$ when $\omega=\sqrt{\left(k_{I *}\right) /\left(k_{D *}+1\right)}$, sharply decreases with respect to $\omega$ when $\omega<\sqrt{\left(k_{I *}\right) /\left(k_{D *}+1\right)}$, and sharply increases with respect to $\omega$ when $\omega>\sqrt{\left(k_{I *}\right) /\left(k_{D *}+1\right)}$. Hence, $(1) /\left(\left|\delta_{*}(j \omega)\right|\right) \ll 1$ can be guaranteed by choosing large $k_{P *}$, proper $k_{I *}$, and $k_{D *}$. In this case, the output resulted by the ocean current disturbance $N_{*}(s)$ is minimal in the designed dy-

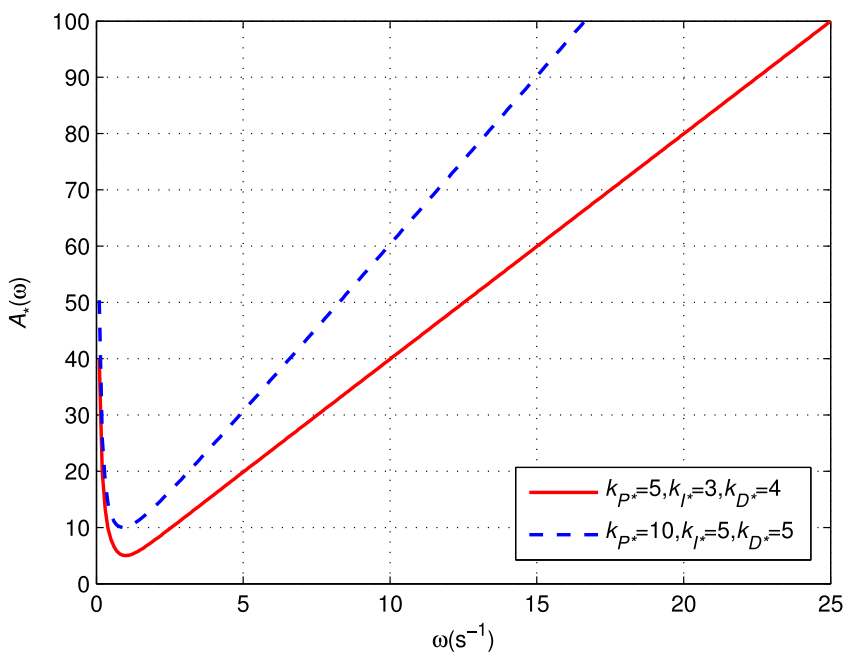

Fig. 11. Amplitude-frequency characteristics of $\delta(s)$.

TABLE III

HYDRODYNAMIC PARAMETERS OF THIS AUV

\begin{tabular}{lcc}
\hline \hline Parameter & Value & Unit \\
\hline$L$ & 1.5 & $\mathrm{~m}$ \\
$m_{11}$ & 1116 & $\mathrm{~kg}$ \\
$m_{22}$ & 2133 & $\mathrm{~kg}$ \\
$m_{33}$ & 4061 & $\mathrm{~kg} \cdot \mathrm{m}^{2}$ \\
$d_{11}$ & 25.5 & $\mathrm{~kg} \cdot \mathrm{s}^{-1}$ \\
$d_{22}$ & 138 & $\mathrm{~kg} \cdot \mathrm{s}^{-1}$ \\
$d_{33}$ & 490 & $\mathrm{~kg} \cdot \mathrm{m}^{2} \cdot \mathrm{s}^{-1}$ \\
$d_{u 2}$ & 0 & $\mathrm{~kg} \cdot \mathrm{m}^{-1}$ \\
$d_{u 3}$ & 0 & $\mathrm{~kg} \cdot \mathrm{m}^{-2} \cdot \mathrm{s}$ \\
$d_{v 2}$ & 920.1 & $\mathrm{~kg} \cdot \mathrm{m}^{-1}$ \\
$d_{v 3}$ & 750 & $\mathrm{~kg} \cdot \mathrm{m}^{-2} \cdot \mathrm{s}$ \\
$d_{r 2}$ & 0 & $\mathrm{~kg}$ \\
$d_{r 3}$ & 0 & $\mathrm{~kg} \cdot \mathrm{s}$ \\
\hline \hline
\end{tabular}

namic control law, which is instrumental in the cable tracking control in the presence of time-varying ocean currents.

\section{NUMERICAL STUDY}

To illustrate the performance of the proposed antinoise cable localization and robust tracking control framework, two numerical examples are carried out using an AUV with a length of $5.56 \mathrm{~m}$ and a mass of $1089.8 \mathrm{~kg}$ in [37]. Its hydrodynamic parameters are listed in Table III, and the maximum propeller thrust and yaw moment are assumed to be $200 \mathrm{~N}$ and $800 \mathrm{~N} \cdot \mathrm{m}$, respectively. Here, the first example is used to evaluate whether the proposed antinoise cable localization method can reduce the negative effects of sensor noise or not. The second example is to validate the performance of the whole cable localization and tracking control framework in the presence of sensor noise and time-varying ocean currents. In the simulation, the control gains are $k_{1}=7.5 \times 10^{7}, k_{2}=1, k_{3}=1.2 \times 10^{9}$, $k_{P u}=0.85, k_{I u}=0.8, k_{D u}=0.75, k_{P r}=0.08, k_{I r}=1.9$, and $k_{D r}=0.50$. 
TABLE IV

Two Methods of CABLE TRACKING

\begin{tabular}{lcc}
\hline \hline No. & Method & Equations \\
\hline 1 & M1 & $(16),(28),(30)$ \\
2 & M2 & $(26),(28),(30)$ \\
\hline \hline
\end{tabular}

TABLE V

Performance Comparison of Two Methods

\begin{tabular}{lccc}
\hline \hline Method & Spike & $\psi_{e, \max } / \mathrm{rad}$ & Control inputs \\
\hline M1 & Yes & 1.76 & Chattering \\
M2 & No & 0.90 & Slight oscillation \\
\hline \hline
\end{tabular}

\section{A. Example 1: Antinoise Evaluation}

In this example, assume that the complicated noise signal shows periodic variation. It is well known that any complicated periodic signal can be expanded in a series of simple sine and cosine functions by Fourier transformation. For instance, the frequencies are composed of $0.5,5,10,15,20, \ldots(\mathrm{Hz})$. To reduce the noise and improve SNR, a proper narrow bandpass filter is often used to remove the high-frequency portion of raw noise. Therefore, we can consider the low-frequency portion of raw noise and in the simulation $f_{\text {noi1 }}$ and $f_{\text {noi2 } 2}$ are chosen as

$$
f_{\text {noi1 }}=f_{\text {noi2 }}=[0.02 \cos (3.14 t)+0.2 \sin (0.1 t)]\left[\begin{array}{lll}
1 & 1 & 1
\end{array}\right]^{\top}(\mathrm{nT}) \text {. }
$$

The symbol ' $\mathrm{T}$ ' denotes the transpose.

Note that the noise magnitude in (38) is almost $0.2 \mathrm{nT}$, and the resulted SNR is almost 20, such that the noise level in the numerical study is the same as that of the field data in [2].

Two methods of calculating the horizontal offset are formulated in Sections III and IV, respectively, which correspond to two kinds of cable tracking methods as listed in Table IV. The first method called M1 represents the classic cable tracking controller based on the accurate magnetic measurement, and the second one called M2 denotes the proposed antinoise cable tracking controller based on the magnetic localization estimation.

The cable tracking results of two methods are shown in Figs. $12-15$ and Table V. Obviously, the first method based on the accurate magnetic measurement in (16) renders a spike signal of the horizontal offset as shown in Fig. 12, which results in jittering behaviors in the transition of the tracking path as shown in Fig. 13 and a large course deviation as shown in Fig. 14. Yet, the cable tracking path of the second method that depends on the proposed antinoise nonlinear estimation in (26) is smooth, which indicates that this dedicated magnetic LOS guidance integrated with magnetic localization estimation is instrumental in antinoise. As shown in Fig. 15, the control inputs of the first method change violently in the initial stage and jitters back and forth in the cable tracking period, whereas the second method reduces this oscillation. It is clear that the second method outperforms the first method in the presence of sensor noise. To know the reason of slight oscillation, we consider the

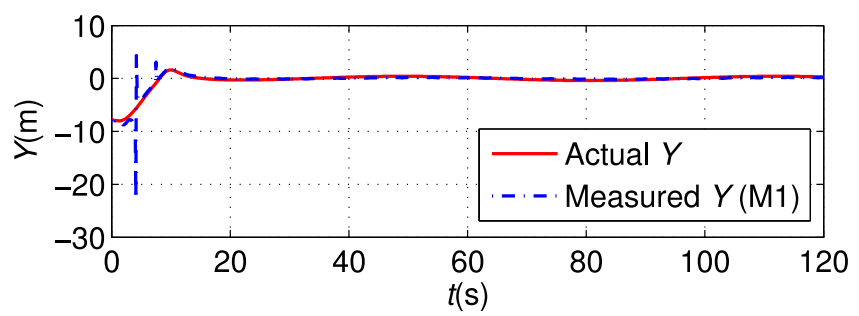

(a)

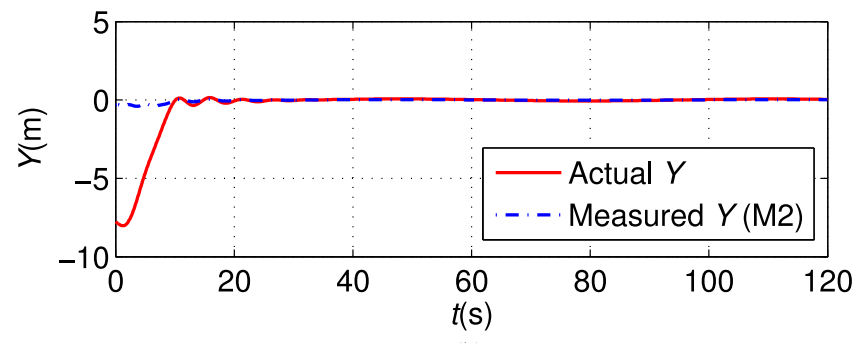

(b)

Fig. 12. Comparison of the horizontal offsets in the presence of sensor noise.

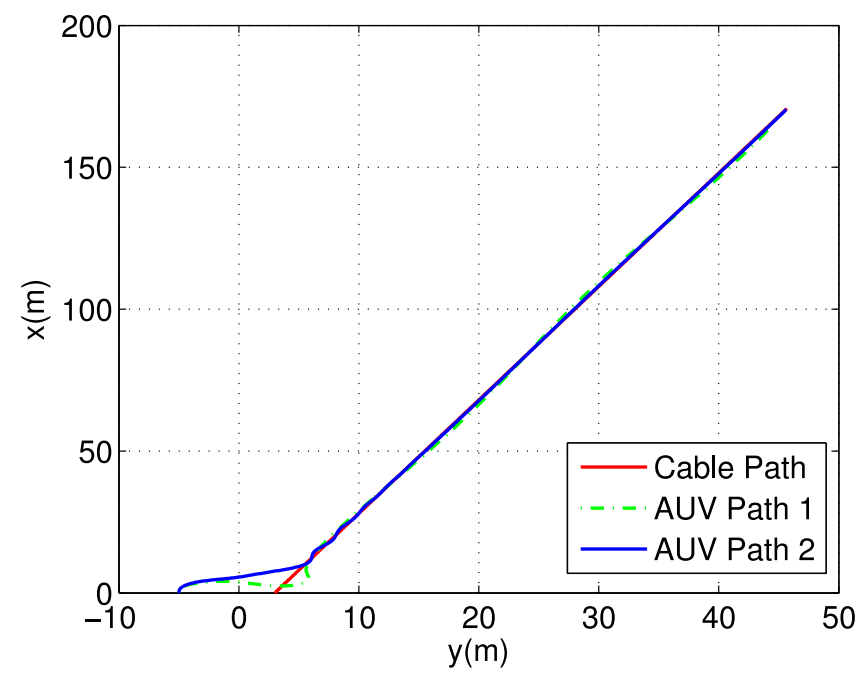

Fig. 13. Comparison of the cable tracking paths in the presence of sensor noise.

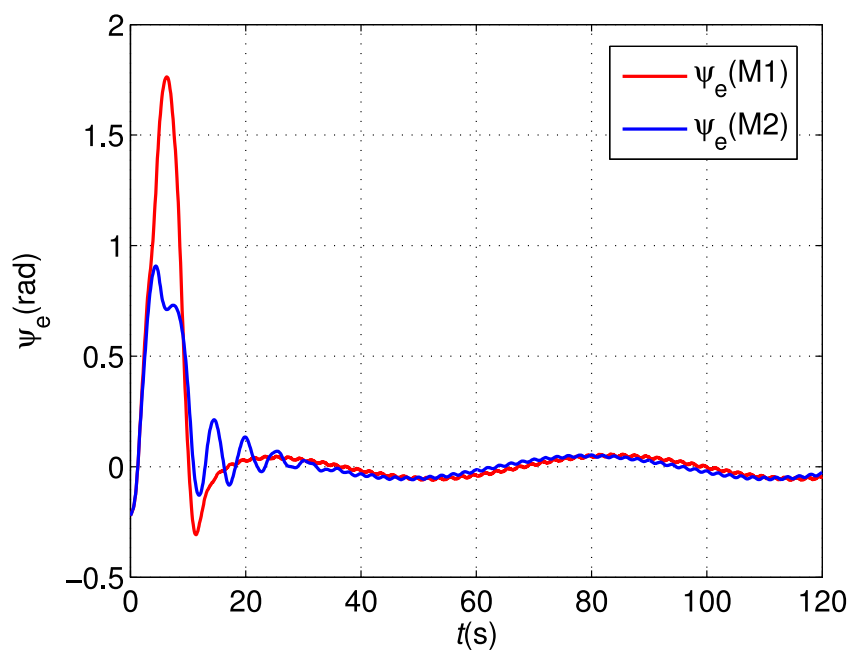

Fig. 14. Comparison of the course deviations in the presence of sensor noise. 

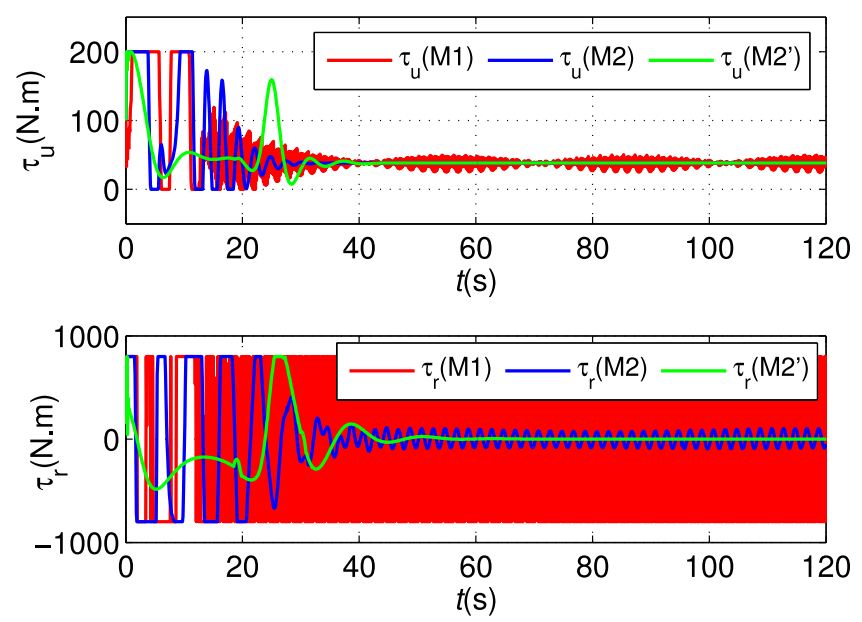

Fig. 15. Comparison of the control inputs without/in the presence of sensor noise.

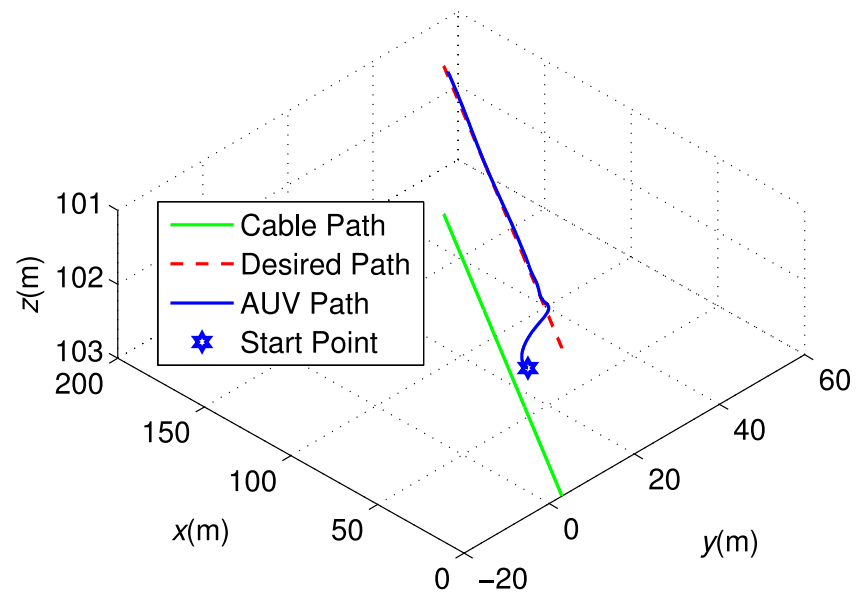

Fig. 16. Subsea cable tracking paths in the presence of sensor noise and ocean currents.
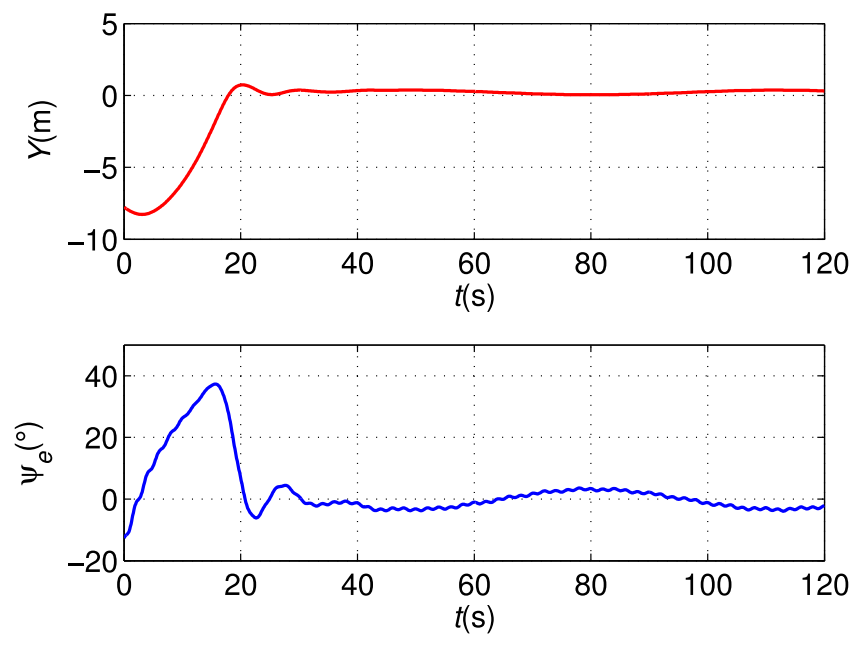

Fig. 17. Subsea cable tracking errors in the presence of sensor noise and ocean currents.
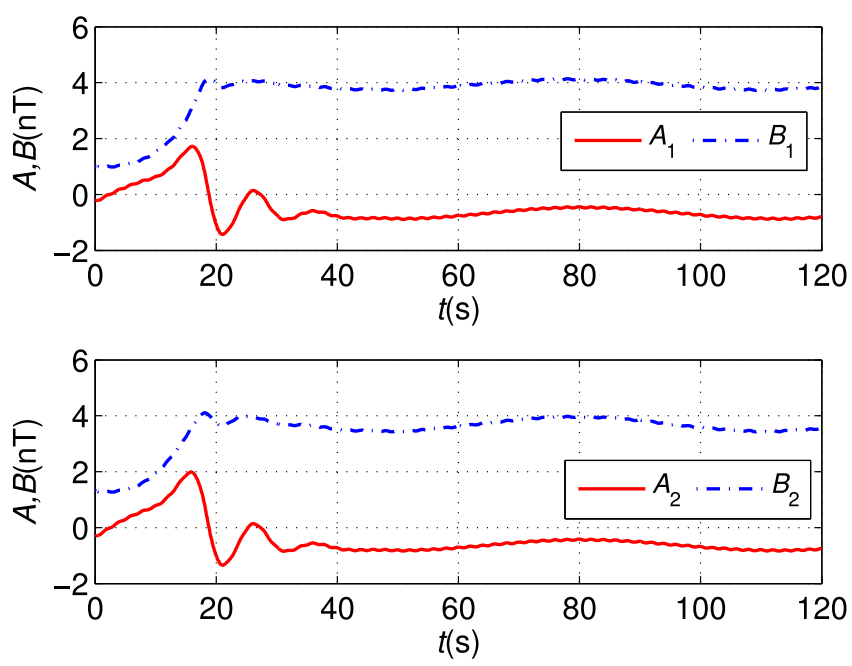

Fig. 18. Transition of the magnetic fields on the magnetometers.
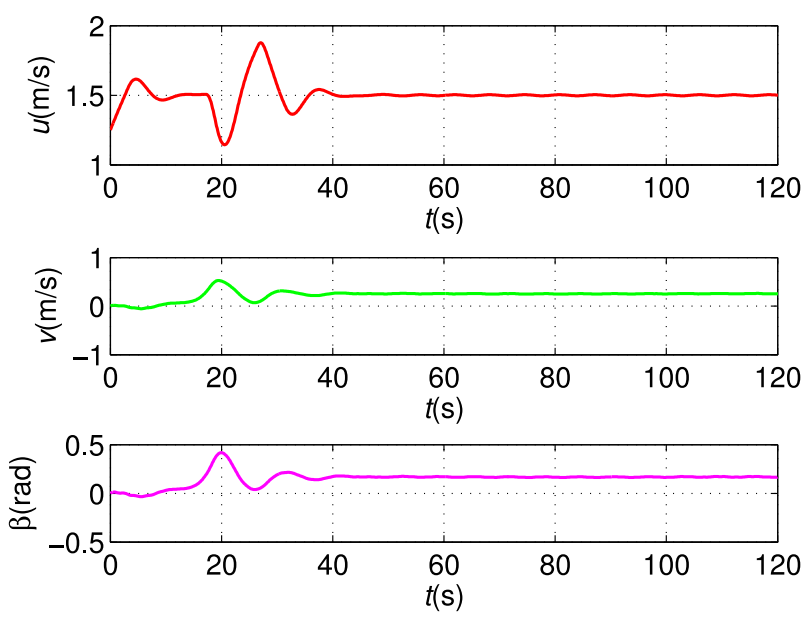

Fig. 19. Transition of the linear speeds and side-slip angle of this AUV.

cable tracking without magnetic noise. The green line describes the corresponding control inputs and there is no oscillation in the transition, which illustrates that the slight oscillation of control inputs using the proposed method (M2) results form magnetic noise rather than the choice of control parameters.

\section{B. Example 2: Robustness Illustration}

In this example, the same AUV is exposed to sensor noise in (38) and time-varying ocean currents as [37]

$$
\left\{\begin{array}{l}
\tau_{E u}=-0.05 m_{11} d(t) \\
\tau_{E v}=0.05 m_{22} d(t) \\
\tau_{E r}=-0.05 m_{33} d(t)
\end{array}\right.
$$

with $d(t)=1+0.1 \sin (t)$.

While accomplishing this cable tracking mission, the AUV is first required to dive to a fixed-depth plane and then move in a given direction obtained from the cable laying report. Assume that itsposition and posture are $[x(0), y(0), \psi(0)]^{\top}=$ $[0 \mathrm{~m},-5 \mathrm{~m},-0 \mathrm{rad}]^{\top}$ when the AUV detects the enough 

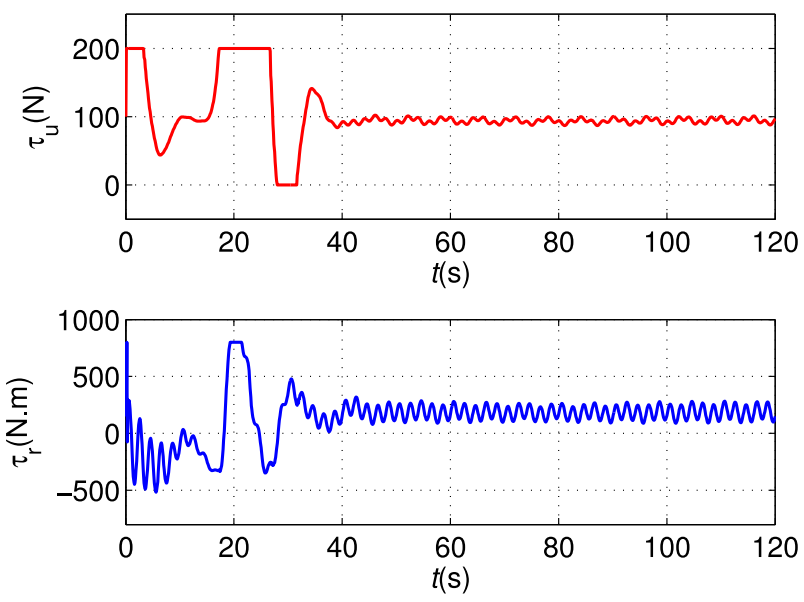

Fig. 20. Transition of the control inputs acting on this AUV.

magnetic field and enters the cable tracking phase, and its speeds are $[u(0), v(0), r(0)]^{\top}=[1.25 \mathrm{~m} / \mathrm{s}, 0 \mathrm{~m} / \mathrm{s}, 0 \mathrm{rad} / \mathrm{s}]^{\top}$. The corresponding cable tracking paths are described in Fig. 16. It shows that the AUV exposed to sensor noise and time-varying ocean currents still moves toward, converges to, and finally follows the desired red dotted path, which is the projection line of the green cable in the above fixed-depth plane.

As shown in Fig. 17, both the horizontal offset $Y$ and the course deviation $\psi_{e}$ reduce to a small neighborhood of zero in the presence of sensor noise and ocean currents, which illustrates that control objectives in (3) have been achieved; namely this AUV follows the cable route with its resultant speed aligned with the direction of the straight cable.

The transitions of the magnetic fields in the tracking period are described in Fig. 18, where both the components along the $x$-axis of magnetometer 1 and magnetometer 2 tend to $-0.6 \mathrm{nT}$ and the components along the $y$-axis tend to $4 \mathrm{nT}$. It means that the yaw angle is not aligned with the direction of the cable and the sway speed is nonzero. As depicted in Fig. 19, this AUV reaches the desired surge speed of $1.5 \mathrm{~m} / \mathrm{s}$, but its sway speed is nonzero and equal to $0.25 \mathrm{~m} / \mathrm{s}$ due to the side-slip effect resulted by ocean currents while tracking a straight-line cable. Obviously, the side-slip angle is not zero, which means that it cannot be ignored in the control design for an underactuated AUV.

The available control inputs of this AUV including the surge force and the yaw moment are depicted in Fig. 20, which keep changing in the straight cable tracking to account for sensor noise and time-varying ocean currents.

\section{CONCLUSION}

In this paper, a two-layer framework is designed to address the problem of robust cable tracking in the presence of sensor noise and ocean currents. The two-layer framework is composed of an antinoise cable localization method in the kinematic layer and a robust tracking control algorithm in the dynamic layer. In the kinematic layer, a simplified magnetic LOS guidance integrated with magnetic localization estimation is proposed to reduce the negative effects of sensor noise. In the dynamic layer, a simpli- fied yet robust feedback linearizing PID controller with reduced implementation complexity is designed to robustly track the desired magnetic guidance profiles. Moreover, the capability of the designed dynamic control laws accounting for ocean currents is analyzed in amplitude-frequency domain. Finally, numerical study illustrates that an AUV using the proposed two-layer framework is able to track subsea cables in the presence of sensor noise and ocean currents. In future work, we expect to focus on extending this method to three-dimensional cable tracking, which should take into consideration the pitch and roll effects of an AUV.

\section{REFERENCES}

[1] T. Szyrowski, S. K. Sharma, R. Sutton, and G. A. Kennedy, "Subsea cable tracking in an uncertain environment using particle filters," J. Marine Eng. Technol., vol. 14, no. 1, pp. 1-13, 2015.

[2] K. Asakawa et al., "Design concept and experimental results of the autonomous underwater vehicle aqua explorer 2 for the inspection of underwater cables," Adv. Robot., vol. 16, no. 1, pp. 27-42, 2002.

[3] X. Sun et al., "Operation-state monitoring and energization-status identification for underground power cables by magnetic field sensing," IEEE Sensors J., vol. 13, no. 11, pp. 4527-4533, Nov. 2013.

[4] X. Xiang, B. Jouvencel, and O. Parodi, "Coordinated formation control of multiple autonomous underwater vehicles for pipeline inspection," Int. J. Adv. Robot. Syst., vol. 7, no. 1, pp. 75-84, 2010.

[5] M. Bibuli, A. Pascoal, P. Ridao, and E. Zereik, "Introduction to the special section on navigation, control, and sensing in the marine environment," Annu. Rev. Control, no. 40, pp. 127-128, 2015.

[6] F. Zhang, G. Marani, R. N. Smith, and H. T. Choi, "Future trends in marine robotics," IEEE Robot. Autom. Mag., vol. 22, no. 1, pp. 14-122, Mar. 2015.

[7] X. Xiang, C. Yu, and Q. Zhang, "On intelligent risk analysis and critical decision of underwater robotic vehicle," Ocean Eng., vol. 140, pp. 453 465, 2017.

[8] Y. Ito, N. Kato, J. Kojima, S. Takagi, K. Asakawa, and Y. Shirasaki, "Cable tracking for autonomous underwater vehicle," in Proc. Symp. Autonom. Underwater Veh. Technol., 1994, pp. 218-224.

[9] J. Kojima, Y. Kato, K. Asakawa, and N. Kato, "Experimental results of autonomous underwater vehicle 'AQUA EXPLORER 2' for inspection of underwater cables," in Proc. OCEANS Conf., 1998, pp. 113-117.

[10] A. P. Aguiar and A. M. Pascoal, "Dynamic positioning and way-point tracking of underactuated AUVs in the presence of ocean currents," Int. J. Control, vol. 80, no. 7, pp. 1092-1108, 2007.

[11] M. Malisoff and F. Zhang, "Adaptive control for planar curve tracking under controller uncertainty," Automatica, vol. 49, no. 5, pp. 1411-1418, 2013.

[12] G. Bruzzone, M. Bibuli, E. Zereik, A. Ranieri, and M. Caccia, "Cooperative adaptive guidance and control paradigm for marine robots in an emergency ship towing scenario," Int. J. Adapt. Control Signal Process., vol. 31, no. 4, pp. 562-580, 2017.

[13] X. Xiang, C. Yu, and Q. Zhang, "Robust fuzzy 3d path following for autonomous underwater vehicle subject to uncertainties," Comput. Oper. Res., vol. 84, pp. 165-177, 2017.

[14] M. Breivik and T. Fossen, "Principles of guidance-based path following in 2d and 3d," in Proc. 44th IEEE Conf. Decision Control Eur. Control Conf., 2005, pp. 627-634.

[15] T. I. Fossen and A. M. Lekkas, "Direct and indirect adaptive integral line-of-sight path-following controllers for marine craft exposed to ocean currents," Int. J. Adapt. Control Signal Process., vol. 31, no. 4, pp. 445463, 2017.

[16] K. D. Do, J. Pan, and Z. P. Jiang, "Robust and adaptive path following for underactuated autonomous underwater vehicles," Ocean Eng., vol. 31, no. 16, pp. 1967-1997, 2004.

[17] J. Guo, F.-C. Chiu, and C.-C. Huang, "Design of a sliding mode fuzzy controller for the guidance and control of an autonomous underwater vehicle," Ocean Eng., vol. 30, no. 16, pp. 2137-2155, 2003.

[18] L. Lapierre and D. Soetanto, "Nonlinear path-following control of an AUV," Ocean Eng., vol. 34, nos. 11/12, pp. $1734-1744,2007$.

[19] L. Lapierre and B. Jouvencel, "Robust nonlinear path-following control of an AUV," IEEE J. Ocean. Eng., vol. 33, no. 2, pp. 89-102, Apr. 2008. 
[20] X. Xiang, L. Lapierre, and B. Jouvencel, "Smooth transition of AUV motion control: From fully-actuated to underactuated configuration," Robot. Autonom. Syst., vol. 67, pp. 14-22, 2015.

[21] W. Caharija et al., "Integral line-of-sight guidance and control of underactuated marine vehicles: Theory, simulations, and experiments," IEEE Trans. Control Syst. Technol., vol. 24, no. 5, pp. 1623-1642, Sep. 2016.

[22] B. Sun, D. Zhu, and S. X. Yang, "Real-time hybrid design of tracking control and obstacle avoidance for underactuated underwater vehicles," $J$. Intell. Fuzzy Syst., vol. 30, no. 5, pp. 2541-2553, 2016.

[23] Z. Peng, D. Wang, Y. Shi, H. Wang, and W. Wang, "Containment control of networked autonomous underwater vehicles with model uncertainty and ocean disturbances guided by multiple leaders," Inf. Sci., vol. 316, pp. 163-179, 2015.

[24] J. Gao, A. A. Proctor, Y. Shi, and C. Bradley, "Hierarchical model predictive image-based visual servoing of underwater vehicles with adaptive neural network dynamic control," IEEE Trans. Cybern., vol. 46, no. 10, pp. 2323-2334, Oct. 2016.

[25] N. Wang and M. J. Er, "Self-constructing adaptive robust fuzzy neural tracking control of surface vehicles with uncertainties and unknown disturbances," IEEE Trans. Control Syst. Technol., vol. 23, no. 3, pp. 991-1002, May 2015.

[26] S. Zhang, J. Yu, A. Zhang, and F. Zhang, "Spiraling motion of underwater gliders: Modeling, analysis, and experimental results," Ocean Eng., vol. 60, pp. 1-13, 2013.

[27] Z. Peng, D. Wang, Z. Chen, and X. Hu, "Adaptive dynamic surface control for formations of autonomous surface vehicles with uncertain dynamics," IEEE Trans. Control Syst. Technol., vol. 21, no. 2, pp. 513-520, Mar. 2013.

[28] C. Liu, J. Sun, and Z. Zou, "Integrated line of sight and model predictive control for path following and roll motion control using rudder," J. Ship Res., vol. 59, no. 2, pp. 99-112, 2015.

[29] Z. Chu, D. Zhu, and S. X. Yang, "Observer-based adaptive neural network trajectory tracking control for remotely operated vehicle," IEEE Trans. Neural Netw. Learn. Syst., vol. 28, no. 7, pp. 1633-1645, Jul. 2017.

[30] N. Wang, M. J. Er, J. C. Sun, and Y. C. Liu, "Adaptive robust online constructive fuzzy control of a complex surface vehicle system," IEEE Trans. Cybern., vol. 46, no. 7, pp. 1511-1523, Jul. 2016.

[31] K. D. Do, "Global path-following control of stochastic underactuated ships: A level curve approach," J. Dyn. Syst. Meas. Control, vol. 137, no. 7, 2015, Art. no. 071010.

[32] T. I. Fossen, K. Y. Pettersen, and R. Galeazzi, "Line-of-sight path following for dubins paths with adaptive sideslip compensation of drift forces," IEEE Trans. Control Syst. Technol., vol. 23, no. 2, pp. 820-827, Mar. 2015.

[33] L. Liu, D. Wang, and Z. Peng, "ESO-based line-of-sight guidance law for path following of underactuated marine surface vehicles with exact sideslip compensation," IEEE J. Ocean. Eng., vol. 42, no. 2, pp. 477-487, 2017.

[34] Z. Zheng, Y. Huang, L. Xie, and B. Zhu, "Adaptive trajectory tracking control of a fully actuated surface vessel with asymmetrically constrained input and output," IEEE Trans. Control Syst. Technol., 2017, doi: 10.1109/TCST.2017.2728518. [Online]. Available: http://ieeexplore.ieee.org/abstract/document/7999258/

[35] X. Xiang, C. Yu, Z. Niu, and Q. Zhang, "Subsea cable tracking by autonomous underwater vehicle with magnetic sensing guidance," Sensors, vol. 16, no. 8, 2016, Art. no. 1335. [Online]. Available: http://www.mdpi.com/1424-8220/16/8/1335

[36] T. I. Fossen, Handbook of Marine Craft Hydrodynamics and Motion Control. New York, NY, USA: Wiley, 2009, ch 2.

[37] K. D. Do and J. Pan, Control of Ships and Underwater Vehicles: Design for Underactuated and Nonlinear Marine Systems. Berlin, Germany: Springer-Verlag, 2009, ch 12.

[38] M. Caccia, M. Bibuli, R. Bono, and G. Bruzzone, "Basic navigation, guidance and control of an unmanned surface vehicle," Autonom. Robots, vol. 25 , no. 4 , pp. $349-365,2008$.

[39] W. S. Levine, The Control Handbook. Boca Raton, FL, USA: CRC Press, 1996, ch. 2.

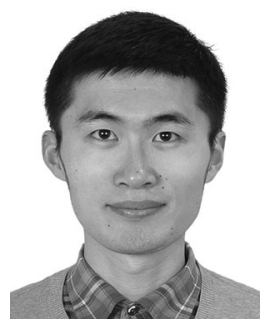

Caoyang Yu received the B.E. degree in marine engineering from the School of Naval Architecture and Ocean Engineering, Huazhong University of Science and Technology, Wuhan, China, in 2013, where he is currently working toward the Ph.D. degree in marine engineering.

His current research focuses on the guidance and control of marine robotic vehicles.

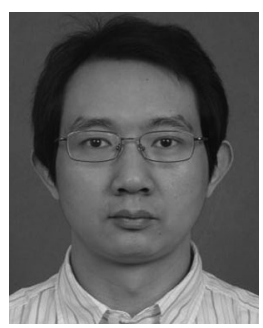

Xianbo Xiang received the B.E. and M.E. degrees in automatic control and marine engineering from Huazhong University of Science and Technology, Wuhan, China, in 2000 and 2003, respectively. and the $\mathrm{Ph} . \mathrm{D}$. degree in robotics from the University of Montpellier 2, Montpellier, France, in 2011.

He was a Lecturer at Huazhong University of Science and Technology. From September 2006 to December 2006, he was an EU Erasmus Mundus Visiting Scholar in the SpaceMaster Project. From February 2008 to March 2011, he was in the European Project FreeSubNet as an EC Marie Curie ESR Fellow at Laboratoire d'Informatique, de Robotique et de Microelectronique de Montpellier, CNRS UMR 5506, Montpellier, France. He is currently an Associate Professor in the School of Naval Architecture and Ocean Engineering, Huazhong University of Science and Technology. His research interests include robotics and marine vehicle systems.

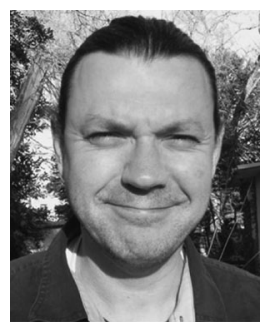

Lionel Lapierre received the Ph.D. degree in robotics from the University of Montpellier 2, Montpellier, France, in 1999.

He then joined the team of Prof. A. Pascoal within the European Project FreeSub for three years in Instituto Superior Técnico, Lisbon, Portugal. Since 2003, he has been in the Robotics Department, Laboratoire d'Informatique, de Robotique et de Microelectronique de Montpellier, CNRS UMR 5506, Montpellier, France. He is currently an Associate Professor at the University of Montpellier 2.

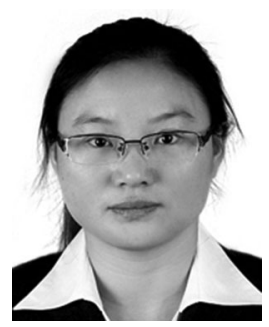

Qin Zhang received the B.S. degree in mechanical engineering from Northeast Petroleum University, Daqing, China, in 2000, the M.Sc. degree in marine engineering from Huazhong University of Science and Technology, Wuhan, China, in 2003, and the Ph.D. degree in robotics from the University of Montpellier 2, Montpellier, France, in 2011.

From January 2012 to March 2012, she was a Postdoctoral Fellow in the DEMAR Project, INRIA Sophia Antipolis. Since May 2012, she has been a JSPS Research Fellow affiliated with the Department of Mechanical Systems Engineering, Tokyo University of Agriculture and Technology. She is currently an Associate Professor in the State Key Laboratory of Digital Manufacturing, Equipment and Technology, Huazhong University of Science and Technology. Her research interests include control of biomechanical systems and robotic systems. 